\title{
Video Streaming in Urban Vehicular Environments: Junction-Aware Multipath Approach
}

\author{
Ahmed Aliyu ${ }^{1,2}{ }^{(\mathbb{D}}$, Hesham El-Sayed ${ }^{3}{ }^{(0)}$, Abdul Hanan Abdullah ${ }^{1}$, Intyaz Alam ${ }^{4}$, Jun Li ${ }^{5}$ and \\ Mukesh Prasad ${ }^{5, *}$ \\ 1 Faculty of Computing, Universiti Teknologi Malaysia, Johor Bahru 81310, Malaysia; \\ ahmedaliyu8513@gmail.com (A.A.); hanan@utm.my (A.H.A.) \\ 2 Department of Mathematics, Faculty of Science, Bauchi State University Gadau, Bauchi State 751105, Nigeria \\ 3 College of Information Technology, UAE University, Al Ain 15551, UAE; helsayed@uaeu.ac.ae \\ 4 School of Computer \& Systems Sciences, Jawaharlal Nehru University, New Delhi 110067, India; \\ intyaz62_scs@jnu.ac.in \\ 5 School of Computer Science, FEIT, University of Technology Sydney, Sydney 2007, Australia; \\ jun.li@uts.edu.au \\ * Correspondence: mukesh.nctu@gmail.com
}

Received: 1 July 2019; Accepted: 7 October 2019; Published: 30 October 2019

\begin{abstract}
In multipath video streaming transmission, the selection of the best vehicle for video packet forwarding considering the junction area is a challenging task due to the several diversions in the junction area. The vehicles in the junction area change direction based on the different diversions, which lead to video packet drop. In the existing works, the explicit consideration of different positions in the junction areas has not been considered for forwarding vehicle selection. To address the aforementioned challenges, a Junction-Aware vehicle selection for Multipath Video Streaming (JA-MVS) scheme has been proposed. The JA-MVS scheme considers three different cases in the junction area including the vehicle after the junction, before the junction and inside the junction area, with an evaluation of the vehicle signal strength based on the signal to interference plus noise ratio (SINR), which is based on the multipath data forwarding concept using greedy-based geographic routing. The performance of the proposed scheme is evaluated based on the Packet Loss Ratio (PLR), Structural Similarity Index (SSIM) and End-to-End Delay (E2ED) metrics. The JA-MVS is compared against two baseline schemes, Junction-Based Multipath Source Routing (JMSR) and the Adaptive Multipath geographic routing for Video Transmission (AMVT), in urban Vehicular Ad-Hoc Networks (VANETs).
\end{abstract}

Keywords: junction-aware; VANETs; multimedia; video streaming; multipath; vehicular network; next forwarding vehicle; vehicle selection

\section{Introduction}

Wireless communication between moving vehicles is increasingly becoming the focus of research for both automobile companies and academic research communities [1-3]. It is driven by the vision that the exchange of information between vehicles can be exploited to improve the safety and comfort of drivers while in motion [4-6]. One of the most valuable Vehicular Ad-Hoc Networks (VANETs) applications is video streaming $[7,8]$. Which can offer more understandable and attractive on-road traffic information to drivers and passengers $[9,10]$. The communicated video can be related to driving safety; for example, an accident ahead, or pedestrians or animals crossing the road [11,12]. It can also be related to onboard communications, such as Vehicle-to-Vehicle (V2V) or Vehicle-to-Office (V2O) video conferencing $[13,14]$. On-board infotainment can also offer advertisements provided 
by supermarkets or shopping malls along the road utilizing roadside unit Internet of Things (IoT) environments $[15,16]$. Consequently, the video streaming service can reasonably improve the onboard experience of the vehicles. The daunting issues of video streaming in VANETs are packet drop, delay and bandwidth-constrained wireless network environments $[17,18]$. This is due to the extremely dynamic topology of vehicles and the large size of video data packets sent across the wireless network [19]. These challenges become worse for high-quality video streaming, because of the even larger size of video data $[20,21]$.

Current multimedia applications require high capacity communication links to fecilitate high speed data transfer rate. Video applications require stringent video quality requirements including minimum delay, packet drop, and efficient bandwidth utilization [22,23]. Hence, there is a need for efficient video data transmission considering the characteristics of both VANETs and video streaming applications. When trying to transmit data efficiently, a path with the necessary resources to meet user requirements should be chosen. On the other hand, in conventional data networks, the routing of data is primarily concerned with an end-to-end connectivity [24,25]. The data network protocol generally represents a network with a metric such as hop-count or delay and uses the shortest path and location algorithm for path and location estimation [26,27]. However, in order to support the quality and delay requirements of video streaming, routing protocols usually need to take into consideration the junction area characteristics when forwarding video data [28]. The consideration of junctions on roads during transmission enhances routing decisions, achieving quality video streaming delivery [29]. A number of multipath transmission schemes have been suggested in some research studies, but few studies have considered road features such as road junctions in hop-by-hop transmission. Figure 1 presents vehicles in the junction area. Vehicle A, B and C are the candidate next forwarding vehicles, of which any one can be chosen to forward a video packet. Vehicle $\mathrm{D}$ is the present forwarding vehicle which needs to make a decision regarding which candidate vehicle is suitable for the data packet forwarding. When forwarding a data packet in a junction area, the vehicle after the junction area with the minimum required link quality is considered to be most suitable. Afterward, the vehicle that resides in the junction with the minimum required link quality is considered as the second-best option, while the vehicle before the junction with the minimum required link quality is the third-best option. This is because the vehicle after the junction might be closer to the targeted destination, which gives it longer connectivity and reduces the number of hops that need to be traversed. Some studies use the junction point as a forwarding area without considering the freshness of the direction of the vehicle.

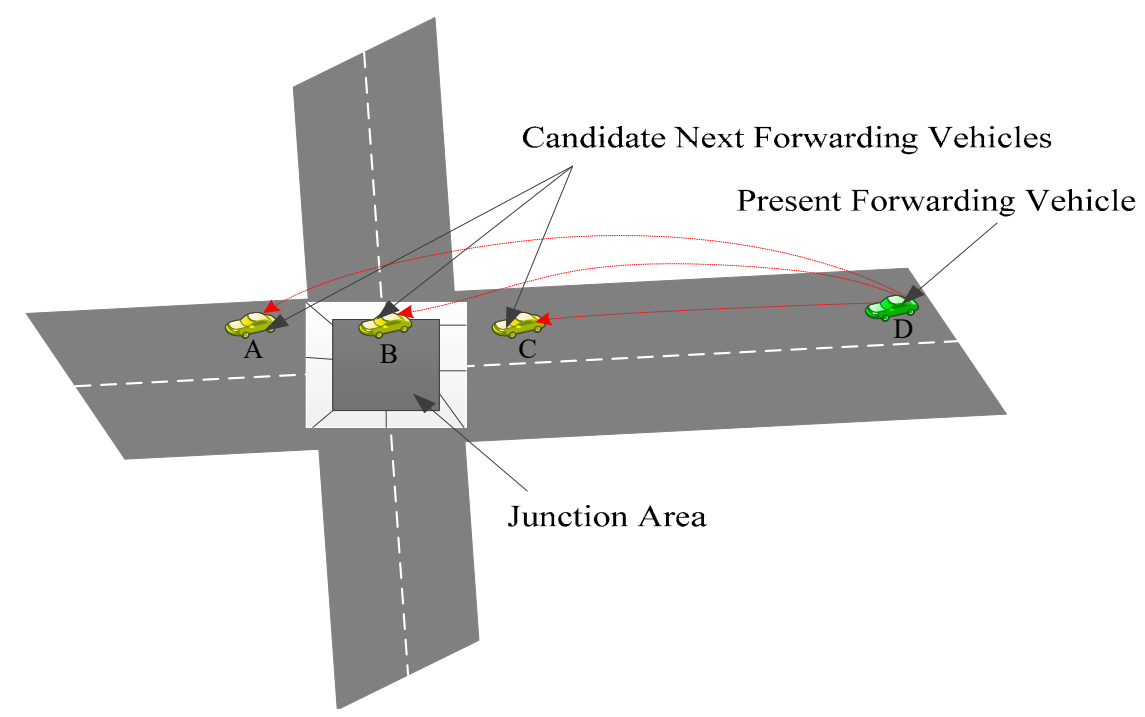

Figure 1. Candidate next forwarding vehicles in the junction area. 
In a junction area, the location and direction of a vehicle are essential when the vehicles' signal coverage extends to a junction area because the selected Next Forwarding Vehicle (NFV) might change its direction of navigation, which could lead to a video packet drop, thereby affecting the quality of the video streaming. Therefore, there is a need to explore the characteristics of the junction area including vehicles that have exited the junction, vehicles in the junction and vehicles before the junction. For the video data, the video frames forwarded are split into three different frames, namely the I-frame, P-frame, and B-frame, which are forwarded via a multipath setting. The I-frames are forwarded through a dedicated path, while both $\mathrm{P}$ and $\mathrm{B}$ frames are forwarded through another path. This idea enables a higher priority for I-frames, which are the most important frames as they interpret both $\mathrm{P}$ and B frames.

In this paper, we proposed a Junction-Aware scheme for Multipath Video Streaming (JA-MVS) considering different points at the junction area in order to avoid or minimize video packet error or drop. Further, mathematical formulations have been adapted to estimate the suitability of a node for data packet delivery. Precisely, the contributions of this study are highlighted as follows:

(1) An enhanced vehicle selection considering the different points in the junction area in order to minimize packet drop due to changes of vehicle direction in the junction area.

(2) Improved vehicle selection based on link quality calculation, considering the signal to interference plus noise ratio (SINR), in order not to select vehicles with high noise due to obstructing objects in the urban environment.

(3) The simulation and performance evaluation of the proposed scheme.

The remaining portions of the paper are organized as follows. In Section 2, a comprehensive review of the related literature is discussed. Section 3 presents the proposed JA-MVS scheme. Section 4 presents the implementation and simulation results with their analysis; and finally, Section 5 concludes the study. The related works based on video streaming in vehicular communication considering regular street and junction area video data forwarding are discussed in the next section.

\section{Related Literature}

In this section, related works in video streaming based on normal street and junction area video data forwarding over a vehicular network have been discussed. Sections 2.1 and 2.2 explains the street-oriented video data forwarding and junction-oriented data forwarding, respectively.

\subsection{Street-Oriented Vehicle Selection}

The normal street-oriented video data forwarding in vehicular communication is discussed considering the different existing work that do not consider the junction area during video forwarding. For example, in Yaqub, et al. [30], a Collaborative Video Retrieval (CoRe) scheme has been proposed in order to address the issue of a bandwidth-constrained cellular network, which affects the quality of a video transmitted by a vehicle. The CoRe scheme enables vehicles to transfer quality video from the Internet and distribute it among other vehicles; that is, neighbor vehicles that reside in a normal street during data forwarding. The collaborating vehicles are selected by taking advantage of their on-road characteristics. These on-road characteristics include their obtainable cellular bandwidth, relative velocity, connection period and Euclidean distance. In addition, the neighbor vehicles, which are the collaborating vehicles, download the video stream via the cellular link and disseminate it to a requesting vehicle based on the Dedicated Short-Range Radio Communication (DSRC) protocol. However, only on-road characteristics have been considered; the junctions' characteristics have not been explored. Further, a Multiple Unicast Path-Forwarding (MUPF) scheme has been proposed to tackle the challenges of the traditional IP-based communication in the vehicular network. The scheme explored Information-Centric Networking (ICN) and numerous unicast forwarding paths for data packet forwarding. In addition, the selection of the routing paths considers the issue of link breakage 
and link quality in relation to the mean response time. However, the situation in which the forwarding vehicle is in the junction area has not been explored.

A cooperating neighbor vehicle solution based on the Game-Theory approach for Platoon-centric (GT4P) driving has been suggested to address the challenges of the short contact time among vehicles during multimedia data transmission, which could lead to delay and video packet error [31]. Thus, the video packet error decreases the Quality of Experience (QoE) of the disseminated video. Therefore, a set of neighbor vehicles, which are navigating in the same direction and are willing to collaborate, forms a platoon member that serves as a forwarding vehicle. The GT4P approach improves collaboration among neighbor vehicles by giving a reward to participating vehicles in the platoon. The collaborating platoon members are formed by considering link quality, travel path and mobility parameters including direction, distance, and speed, which minimizes the effect of vehicle mobility on the video streaming transmission. However, the platoon members at different points in the junction area are not considered. Thus, there is a need to explore the junction characteristics.

Similarly, a comparative analysis for platoon-centric video streaming transmission in autonomous VANETs has been suggested to assess and ascertain the Quality of Experience (QoE) for shared video flows [32]. Different vehicle distances between the source and destination vehicle and various video characteristics are employed. Thus, the effectiveness of the platoon-centric video transmission is justified considering different video metrics. Further, platoon-centric driving offers a collaborative navigation arrangement for a set of vehicles with the same navigation route such that the member vehicles in the platoon keep almost a fixed distance among themselves. In a typical platoon approach, the cruise control system utilizes the onboard sensors—-for example, laser or radar-to estimate the distance between vehicles and then adjust their speed. Further, a member vehicle obtains information from the platoon leader via vehicle-to-vehicle communication. Thus, collision is minimized through communication with vehicles ahead. The platoon approach alleviates the effect of the short contact time that leads to video packet loss or error in the time of video transmission. However, the whole approach does not look into junction-area-based video packet forwarding.

In order to address the issue of high latency in locating a possible content provider in an information-centric network, a Preference-Aware Fast Interest Forwarding (PaFF) method for video streaming has been suggested [33]. In the PaFF approach, each vehicle forms a Highly Preferred Content Table (HPCT) in order to preserve the content catching status of vehicles that have similar video playback behaviors and mobility parameters. Considering the HPCT, a vehicle forwarder that employs a preference mechanism is explored to choose the next hop of relevant video packets to minimize delay and improve reliability. The selection of a potential forwarder vehicle for the content delivery depends on fundamental mechanisms including the estimation of similar mobility parameters of neighbor vehicles, preference agreement and potential vehicle discovery beyond one-hop neighbor vehicles. However, in the mobility parameters for potential forwarding vehicles, the various points at the junction have not been considered. Therefore, the next subsection discusses video transmission considering the junction areas of roads.

\subsection{Junction-Oriented Vehicle Selection}

In this subsection, the existing solutions that consider road junctions during video data forwarding are discussed. In the multipath setup, very few research studies have considered the realistic nature of VANET roads; for example, in $[34,35]$. The intersections and junctions of roads need to be considered to select vehicles for multipath transmission. The incorporation of junctions/intersection into multipath transmission offers more realistic and efficient and higher- quality video streaming. Thus, in Sermpezis et al. [34], an analytical Junction-Centric Multipath Source Routing mechanism (JMSR) has been suggested. JMSR features include junction-aware logics, the multipath route from the source to destination and the source routing scheme. The JMSR employs geographic routing protocols, meaning that the locations of the junctions of a street are leveraged through the street's digital maps for data forwarding purposes. In the multipath setup, two paths are preserved concurrently considering 
the numerous junctions which a routed data packet has to traverse before reaching its destination. In addition, the JMSR embeds routing details into an individual packet, based on the source routing standard. The source routing standard is set-up in such a way that every individual vehicle in the path knows the route the packet must traverse. However, in JMSR, all vehicles are partitioned or grouped into different routes of paths, and then the cost of each path is calculated before being selected as the path for data forwarding. This approach is prone to high overhead and delay, which is a critical issue in video streaming requirements. In addition, the JMSR updates the information about the position of other vehicles when the position of the destination vehicle changes. Since it is believed that vehicles are moving very quickly, there is a frequent change of position; thus, it is important to consider the different points of the junction area and the direction of the vehicle at the junction. The different points of the junctions that have not been considered include the vehicle after the junction, before the junction, and inside the junction. The vehicle position, road-ID and traffic status can be employed to estimate whether a vehicle has exited the junction and has taken a direction towards the destination vehicle. Thus, video packet loss can be minimized since the most optimal vehicle is considered as a forwarder at the junction area.

In Salkuyeh and Abolhassani [35], an adaptive multipath video streaming method based on geographic routing has been suggested. The adaptive scheme selects multiple paths, depending on the volume and lifetime of the video to be transmitted from the source to destination. The route connection probability has been employed to select the best route. The connection probabilities are divided into two, namely street and junction connection probability, based on the cells and line of sight of the vehicles, respectively. However, the probabilistic connectivity approach does not consider the position and direction of the vehicle for connectivity. In addition, priority is not given to a vehicle that has already exited the junction and which is in the direction of the destination vehicle. This may lead to the selection of an inappropriate next forwarding vehicle in the junction, which can cause packet loss and affect the quality of the video streaming.

De Felice et al. [22] suggested a distributed beaconless routing protocol for pre-recorded video data transmission (DBD) over VANETs. It is an integrated framework that handles the QoE of video services and routing protocols. DBD further advances the performance of the IEEE 802.11p/WAVE MAC layer by resolving the spurious forwarding problem. However, an adaptive backbone mechanism is not considered for the DBD. An opportunistic routing solution for pre-recorded video (ORV) streaming is proposed to handle the interference of wireless fading channels [36]. The mechanism takes into consideration the interference of vehicles from the surroundings during the relay selection procedures. Nevertheless, the SSIM index of the video streams is not considered in measuring the quality of the video. Multiple path solutions with error correction for video streaming over VANETs (LIAITHON+) have been presented; the aim of this is to reduce collision and packet loss in high data rate networks [37]. LIAITHON+ employs a multipath approach to distribute high data rate traffic into a set of paths. However, the quality of the streaming video is not measured based on PSNR and SSIM index metrics. Al-Ani and Seitz [38] present a video stream routing QoS for the multi-rate mechanism in order to achieve congestion control and avoidance. The mechanism employs the Ant Colony Optimization (ACO) algorithm and Simple Network Management Protocol (SNMP) monitoring features. The mechanism is called QoRA; it decides on paths by considering applications that QoS needs and prevents transmission flow from entering congested nodes. Nevertheless, the mechanism is not adequately benchmarked. QoE-driven and link-quality receiver-based (QOALITE) transmission is proposed to imrpove the quality of video while considering a challenging VANET environment [39]. A geographical receiver-based beaconless strategy has been proposed as a solution for transmitting video streams in VANETs. However, some parameters are considered in choosing the best relay node and building up reliable backbones to deliver video messages. In addition, the dynamic adjustment of the time window needs to be considered for tackling collisions. Therefore, proper vehicle selection in the junction area is not considered in some routing schemes. Consequently, an optimal next forwarding vehicle selection scheme is required that considers the junctions and neighbor vehicle 
mobility information and link quality to select an optimal next forwarder for video streaming in the multipath setup.

\section{Junction-Aware Vehicle Selection Scheme}

In this section, the proposed Junction-Aware Multipath Video Streaming (JA-MVS) scheme is presented considering the various vehicle positions at the junction area. The multipath transmission considers the junction-aware concept in hop-by-hop transmission. The consideration of road junctions during transmission enhances routing decisions to achieve quality video streaming delivery. A number of multipath transmission schemes have been suggested in some research studies, but few studies have considered road features such as junctions in hop-by-hop transmission. Some of these studies use the junction point as a forwarding area without considering the freshness of the direction of the vehicle. In a junction area, the location and direction of the vehicle are essential when the vehicles' signal coverage extends to a junction area, because the selected NFV might change its direction of navigation, which could lead to a video packet drop, thereby affecting the quality of the video streaming. Therefore, there is a need to explore the characteristics of the junction area including the vehicles exiting the junction, vehicles at the junction and vehicles before the junction. The vehicles before the junction are considered to be the vehicles at the end of the road, which is at the traffic light. The vehicles at the junction do not have a road-ID but might be in the direction of the Destination Vehicle Node (DVN), while the vehicles after the junction have recently changed their road-ID, navigating towards the direction of the DVN. Considering the vehicles at a road junction, three cases have been considered, as stated in the aforementioned discussion.

In the first case, the vehicles which have exited the junction are preferred and considered to be vehicles that have already chosen their direction of navigation. Hence, their direction is known and probably closer to the DVN; hence they are employed as the NFVs. In the second case, a vehicle at the end of the road, which is close to the junction, is the second preferred selection area for NFVs, because a vehicle before the junction might need to wait for a traffic light; thus, its direction does not change instantly and it can be used to forward video streaming to the direction of the DVN. The third case is employed if there is no suitable vehicle that has exited the junction and no vehicle at the end of the road; then, the vehicle inside the junction area is selected. However, the third case is only employed if the first and second cases do not occur. Therefore, in the proposed JA-MVS, the vehicles that have already exited the junction or intersection area are considered based on the freshness of their location, direction and speed information. Consequently, the JA-MVS scheme is in two stages: information exchange and a video streaming data forwarding stage in the junction area. The detailed discussion of the JA-MVS scheme is given in the next subsections.

\subsection{Information Exchange Phase}

At the information collection phase, every vehicle exchanges a hello message with its neighbor vehicle. The hello message (HM) is exchanged within a time interval of every second. The HM content includes the vehicle direction, position, ID, road-ID and hello message time-stamp. The generated hello messages are stored in a Neighbor Information Table (NIT). The NIT is updated in every time interval of the hello message exchange; that is, one second. The parameters in the NIT are recorded based on tuple since the collections of items are different. The position is based on $\mathrm{x}, \mathrm{y}$ coordinates, which are centered on relative distance. The road-ID is simply an identifier which is alphanumeric. The time stamp is recorded in seconds. The direction is based on four cardinal directions including north, south, west and east. The vehicle-ID is recorded based on an alphanumeric identifier. The information packet format is depicted in Figure 2. The direction and position are estimated using the GPS of each vehicle, which is employed to determine the location of the vehicle in the junction area. The road-ID is used to determine if the vehicle is on the new road and is employed for the vehicle selection at the junction area. A linked list is used to store packet information, as the sizes of each element are different, with standard units considered such as milliseconds for time stamps, latitude and longitude with six-digit 
decimal points, etc. In addition, the selection considers the quality of the vehicle signal based on the signal to interference plus noise ratio (SINR), as formulated in Equation (1), before considering the three cases in the junction area. In addition, the vehicle density at the junction has been considered for suitable candidate vehicle selection, which is shown in Equation (2). In the generic concept of the geographic routing, the Source Vehicle Node (SVN) is already conscious of the direction and location of the DVN in the network based on the location service system and GPS. Therefore, the greedy algorithm is adapted in a way that not only selects the vehicle closer to the destination but also takes into account the aforementioned three different cases of the junction area.

$$
\operatorname{SINR}_{\left(p_{i}, p_{j}\right)}=\frac{S_{P W} F_{R D\left(p_{i}, p_{j}\right)} \times l\left(p_{i}-p_{j}\right)}{\varphi+\sigma\left(p_{j}\right)}
$$

where $p_{i}$ is the sender vehicle's position and $p_{j}$ is the signal receiver vehicle's position. Thus, $S_{P W}$ is the transmitting signal strength, and $F_{R D}$ is the random fading between the sender vehicle and the receiving vehicle. In addition, $l$ is the distance between $p_{i}$ and $p_{j}$, while $\varphi$ and $\sigma$ denote the external noise and small short-noise of $p_{j}$, respectively. We have considered one direction; that is, the directions of travel of the sensor and receiver vehicles are the same. Here, it is noteworthy that the proposal will also work for the two-dimensional scenario; that is, the directions of travel of the sender and receiver vehicles could be different or in opposite directions. However, in this case, the computation will be somewhat impacted by the speed of vehicles. Consequently, both the distance and SINR are employed when selecting a vehicle in the junction area. The vehicle density is estimated as

$$
V D R=\frac{(3 \times I D V D)+O D V D}{4 \times 2 H o p}
$$

The next forwarding vehicle is selected based on the Candidate Next Forwarding Vehicle (C-NFV) which has the highest value of Vehicle Density of the Road (VDR). However, the Opposite Direction Vehicle Density (ODVD) is given a smaller value by multiplying it with $\frac{1}{4}$, which is three times lower than the value of the In-Direction Vehicle Density (IDVD) multiplied by $\frac{3}{4}$. This is because the vehicles moving in the opposite direction are considered not to have longer or continuous connectivity with the forwarding vehicle. This ensures that a C-NFV moving towards the direction of the DVN is given higher priority. Thus, a threshold has been assigned as $V D R_{\max }$, where the $V D R_{\max }$ is the estimated highest value of neighbor nodes that does not lead to congestion. Therefore, the $V D R_{\max }$ is set to 100 vehicles $/ \mathrm{km}$. We have considered a road environment with opposite lanes and assumed that 50 vehicles on both sides of opposite lanes in a $1 \mathrm{~km}$ road length will not result in congestion. This will result in a 20-meter road distance for each vehicle, which we consider a normal situation without congestion in urban road environments. The C-NFV case that has a greater number of vehicles than the $V D R_{\max }$ $\left(V D R>V D R_{\max }\right)$ is considered to be a congested network. Therefore, we obtain Equation (3):

$$
V D R= \begin{cases}1 & V D R \leq V D R_{\text {max }} \\ 0 & V D R>V D R_{\text {max }}\end{cases}
$$

The VDR is estimated for two multiple paths independently since the paths are dispersed. The road density of a C-NFV is compared with the other road densities of the neighbor vehicles of a Candidate Next Forwarding Vehicle, and the road with the highest density, but which is not greater than the $V D R_{\max }$, is considered for selection. Therefore, the vehicle density of the road is considered to be one of the important metrics that enables optimal NFV selection. This, in turn, improves the quality of the video transmission, since the faster selection of NFV has been considered based on the density of the vehicles on the road. The most suitable node depends on the two parameters of SINR and VDR, as represented in Equation (4):

$$
S_{n}=\propto\left(M^{\text {SINR }}\right)+\beta\left(V D R_{\text {value }}\right)
$$


where $S_{n}$ represents the suitable node, $M^{S I N R}$ is the maximum SINR value, and $V D R_{\text {value }}$ is the value of the vehicle density of the road. Both $\propto$ and $\beta$ are the weighting factors assigned to each of the parameters. Considering that the SINR guarantees the quality of a link, the $\propto$ is assigned a weight of 0.6 , while the $\beta$ is assigned 0.4 for the vehicle density of the road; thus, the total weight is 1 . Therefore, the link of the suitable node selected is considered to be the most efficient link for video data transmission. Thus, an efficient link can be formulated as represented in Equation (5):

$$
\text { Efficient link }=\frac{T R^{\text {Success }}}{T R^{\text {Total }}}
$$

where $T R^{\text {Success }}$ is the number of packets successfully delivered through a link, and $T R^{T o t a l}$ is the overall attempts performed during data packet transmission.

\section{\begin{tabular}{l|l|l|l|l} 
Position $(\mathrm{x}, \mathrm{y})$ & Road-ID & Timestamp & Direction & Vehicle-ID
\end{tabular}}

Figure 2. Information packet format.

\subsection{Video Data Forwarding Phase}

In this section, the Junction-Aware Multipath Video Streaming concept is discussed considering geographical routing; specifically, the greedy forwarding concept is adapted by modifying some of its concepts. As mentioned in the previous section, firstly, the SINR and VDR of each neighbor vehicle are evaluated based on Equation (1) and (2).

Below mentioned Algorithm 1, presents the procedure involved in video data forwarding when a $\mathrm{C}-\mathrm{NFV}$ is in the junction area. The vehicle mobility information is gathered considering line 2. Line 3 checks if the C-NFV is in the junction area; that is, whether the C-NFV is before the junction, inside the junction or after the junction. Then, the SINR is computed considering the three scenarios of the junction area in line 4. However, the vehicle with the highest SINR value is preferred if it has not exited the junction area, as for the remaining cases of the scenarios. Thus, the SINR value selection has a higher priority than the different positions in the junction area. Lines 5-7 check if the C-NFV's road-ID is ahead of the junction location and if it has a higher signal strength value, and the optimal C-NFV - that is, the RVN-is selected. Lines 8-11 choose the first two RVNs $\left(P_{2}\right.$ and $\left.P_{3}\right)$, whose I-frames are forwarded via $P_{2}$, and the $\mathrm{B}$ and $\mathrm{P}$ frames are forwarded through $P_{3} . P_{2}$ and $P_{3}$ are the first two relay nodes before the other intermediate nodes in the two paths. The suitable RVNs are the nodes with the maximum efficient link, selected using Equation 5. Lines 12 and 13 check if the C-NFV is at the end of the road, which is at the traffic light. This C-NFV is selected if there is no C-NFV after the junction location towards the DVN. Line 14 forwards the video data via the RVN as previously discussed. Otherwise, the C-NFV is checked if its location is inside the junction, meaning a C-NFV with no road-ID, and this is selected if there is no vehicle after the junction or inside the junction based on lines 16-18. Otherwise, the algorithm checks whether the DVN belongs to the set of the C-NFVs: if true, the video data is forwarded to the DVN and terminated; otherwise, it is forwarded to NFV based on lines 19-29. Line 30 terminates the whole procedure of the algorithm. Figure 3 shows the flow of the algorithm. As previously stated, the complete routing concept is based on the greedy-based geographic routing protocol. The implementation process, including the simulation setup and performance evaluation of the proposed JA-MVS scheme, has been presented in Section 4 . 


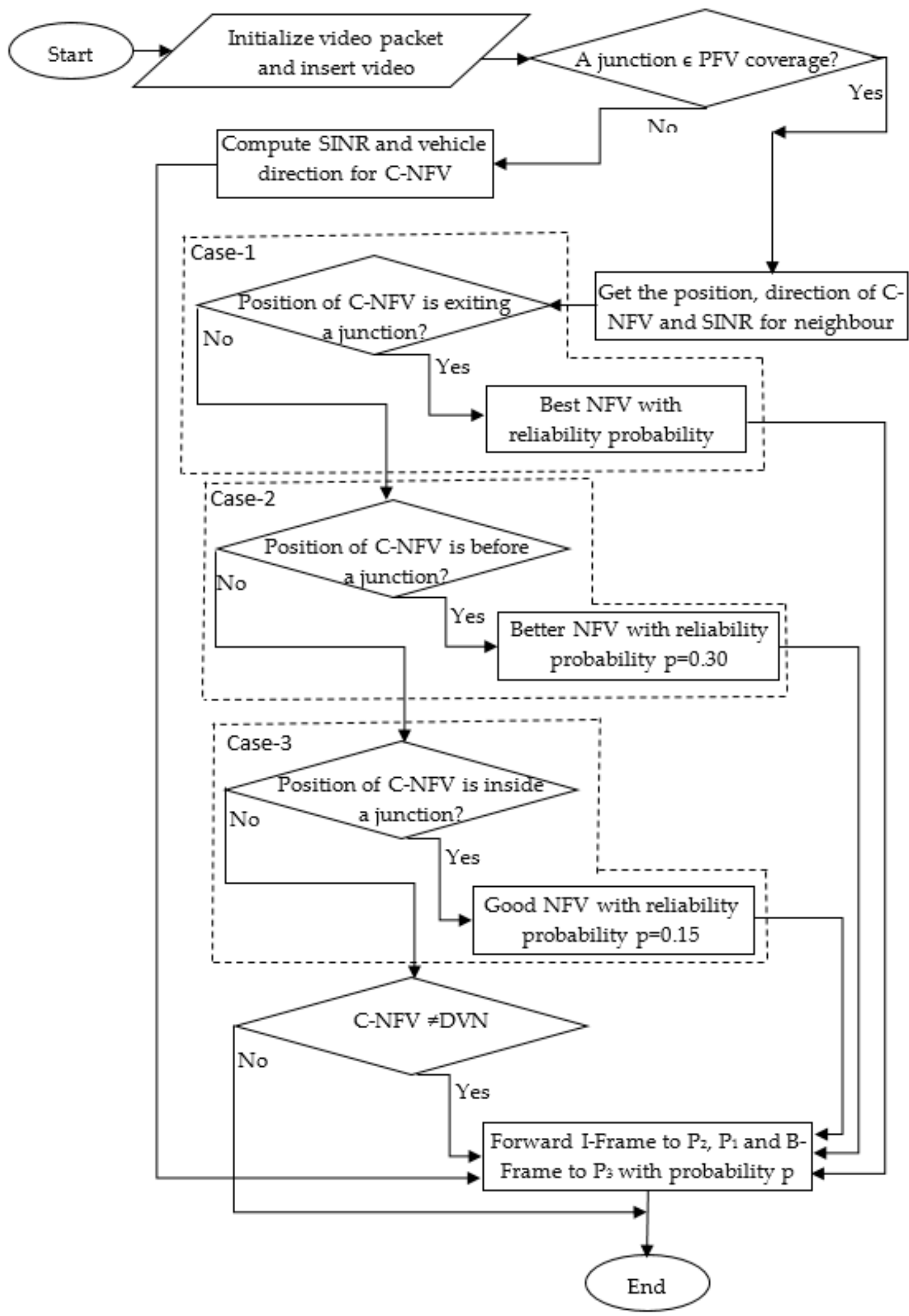

Figure 3. Flowchart for vehicle selection considering the junction area. C-NFV: Candidate Next Forwarding Vehicle; SINR: signal to interference plus noise ratio; DVN: Destination Vehicle Node.

\section{Performance Evaluation}

In this section, the simulation setup results obtained and the performance evaluation with analysis against the baseline schemes are presented. The performance is evaluated considering two different 
cases. each based on vehicle density and video data rates. The evaluation is conducted by considering metrics including the Packet Loss Rate (PLR), Structural Similarity Index (SSIM) and End-to-End Delay (E2ED). The PLR is the ratio of a transmitted video packet to that of the delivered packet. This also helps in ascertaining the quality of the video transmitted, because the lower the number of video packets dropped, the higher the number of video packets delivered at the destination vehicle, which in turn leads to higher video quality [40]; conversely, the higher the number of video packets dropped during transmission, the lower the number of received video packets at the destination vehicle, which leads to lower video quality. The SSIM is computed as the perceived similarity between the transmitted video images and the original video images. The calculation of the SSIM index is grouped into three aspects: contrast, luminance, and structural assessment [41-43]. The E2ED is the total summation of the delay encountered from the source vehicle to the destination vehicle. The delay includes the propagation delay, transmitting delay, processing delay and startup delay [44,45]. The level of E2ED delay also signifies the quality of the video delivered at the destination vehicle. The metrics are tested in relation to different vehicle densities and data rates (kbps). The distribution of the vehicle density is from 50 to 500 , and the video data rate considered ranges from $160 \mathrm{kbps}$ to $1600 \mathrm{kbps}$. The three metrics are utilized considering the different vehicle densities and data rates. Particularly, a high data rate and high vehicle density have been considered. The discussion of the results of the proposed scheme is presented in Section 4. The baseline schemes employed for the benchmarking of the proposed scheme include Junction-Based Multipath Source Routing (JMSR) [34] and Adaptive Multipath geographic routing for Video Transmission (AMVT) in urban VANETs [35]. The simulation of the junction-aware scheme is guaranteed since the whole map of the simulation environment, including roads and junctions, has been integrated using the OSM and integrated into the Simulator of Urban Mobility (SUMO). The vehicles know their positions due to location information service.

The proposed JA-MVS is implemented using the most acceptable network simulators: NS-2.34 and SUMO. The NS-2 is a network simulator that enables the simulation of network communication. The SUMO employs the mobility model generator for VANETs (MOVE). It has the ability to create a realistic model for the mobility of vehicles in urban traffic scenario. The Evalvid has been employed to provide video frames and a video quality evaluation framework. The Manhattan city digital map with a latitude of 39.191 to 39.184 and longitude of -96.574 to -96.563 is employed for the mobility and traffic environment setup (see Figure 4). The digital map structure and data are acquired from the OpenStreetMap contributors.

The details of the simulation parameters are depicted in Table 1, which include the urban simulation area, simulation time, vehicle speed, number of vehicles, the MAC protocol, video resolution, video play duration, the transmission range, frequency bandwidth, propagation model, antenna model, traffic type, channel type, transmission protocol, hello packet timeout and scenarios.

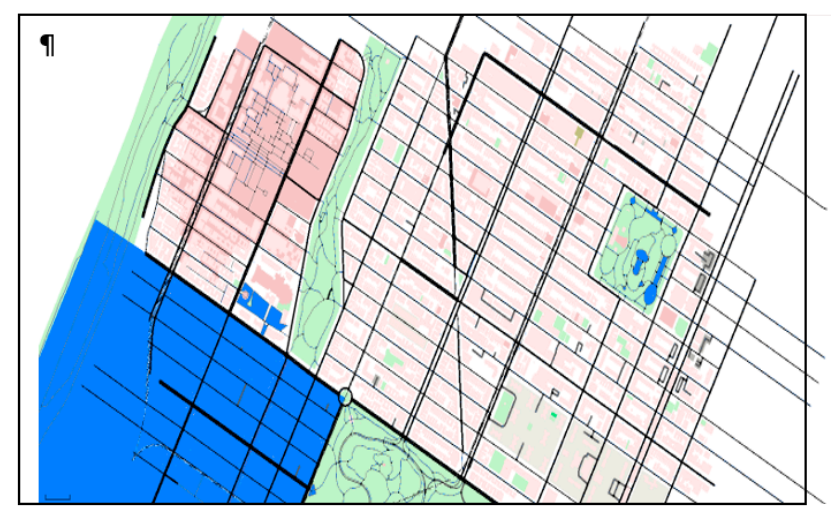

Figure 4. Manhattan city map. 


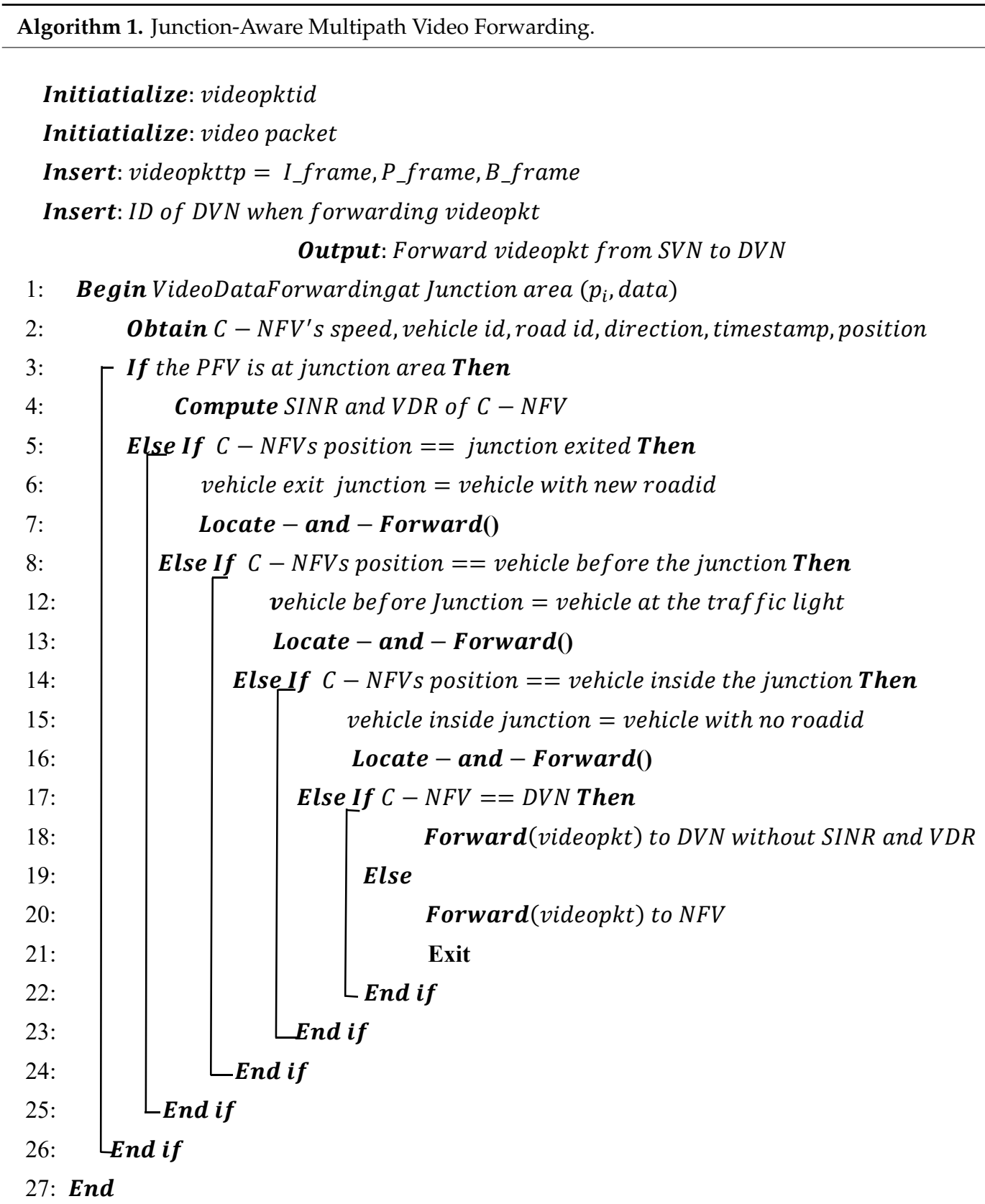

\footnotetext{
Locate-and-Forward ()

1. Identify theNFV with higher SINR and VDR

2. dentify suitable two RVNs from SVN for $P_{2} \& P_{3} \leftrightarrow$ conditions

3. Forward videopkttp $\left(I_{\text {frame }}\right)$ \& videopktid to $N F V$ via $P_{2}$

4. Forward videopkttp( $P_{-}$frame, $B_{-}$frame) to $N F V$ via $P_{3}$
} 
Table 1. Simulation parameters. JMSR: Junction-Based Multipath Source Routing; AMVT: Adaptive Multipath geographic routing for Video Transmission; PLR: Packet Loss Rate; SSIM: Structural Similarity Index; E2ED: End-to-End Delay.

\begin{tabular}{cc}
\hline I. Parameters & II. Values \\
\hline III. Urban simulation area & IV. $1000 \times 1000 \mathrm{~m}^{2}$ \\
\hline V. Simulation time & VI. $600 \mathrm{~s}$ \\
\hline VII. Vehicle speed & VIII. 2.78 to $13.89 \mathrm{~m} / \mathrm{s}(10$ to $50 \mathrm{~km} / \mathrm{h})$ \\
\hline IX. Number of vehicles & X. 50 to 500 \\
\hline XI. MAC protocol & XII. IEEE $802.11 \mathrm{p}$ \\
\hline XIII. Video resolution & XIV. 352 $\times 288$ \\
\hline XV. Video play duration & XVI. 139 s \\
\hline XVII. Transmission range & XVIII. 250 $\mathrm{m}$ \\
\hline XIX. Frequency Bandwidth & XX. 5.9 GHz \\
\hline XXI. Propagation model & XXII. Shadowing \\
\hline XXIII. Antenna model & XXIV. Omni-directional \\
\hline XXV. Traffic type & XXVI. Constant Bit Rate \\
\hline XXVII. Channel type & XXVIII. Wireless \\
\hline XXIX. Transmission Protocol & XXX. UDP \\
\hline XXXI. Hello packet timeout & XXXII. 1 s \\
\hline XXXIII. Scenarios & XXXVI. JMSR and AMVT \\
\hline XXXV. Comparison protocol & XXII. SSIM index and E2ED \\
\hline XXXVII. Metrics &
\end{tabular}

\section{Results Analysis of the JA-MVS Scheme}

The results obtained for the performance analysis of the proposed scheme in comparison against the baseline schemes are presented here. Figures $5 \mathrm{a}$ and $5 \mathrm{~b}$ shows the performance improvement of the JA-MVS scheme against the two baseline schemes of JMSR and AMVT. Figure 5a shows the performance of the schemes based on PLR against different vehicle densities. It is observed during the simulation results that the number of dropped video packets decreases as the vehicle density increases. The two baseline schemes experience more than $40 \%$ video packet drop when the vehicle density is 50; this is due to the consideration of only junctions as nodes in the case of JMSR. The high PLR encountered for the AMVT is related to the building obstruction model used to detect obstructions before sending a video packet to the next forwarding vehicle. The obstruction detection might not be realistic due to the frequent position changes of the vehicle; thus, the vehicle continues carrying packets for some period of time, which are later dropped. However, as the vehicle density increases, there is a higher number of vehicles to be selected as NFVs with better link quality for video packet transmission. Further, with higher density, the two baseline schemes have lower PLR values, which are below $10 \%$, even trending towards 5\%. Nevertheless, the proposed JA-MVS scheme performs better at $7.5 \%$ PLR at 50 numbers of vehicles, with the PLR becoming lower than $5 \%$ when the vehicle density is 150 . The better performance of JA-MVS is connected to the consideration of junctions, which is based on three different cases: the selection of vehicle that has exited the junction, that is before the junction and inside the junction, with its navigation being towards the direction of the DVN. With the aforementioned considerations, the proposed scheme outperforms the two baseline schemes. The average percentage packet losses experienced by JMSR, AMVT, and JA-MVS are $16.3 \%, 14.4 \%$, and $3.4 \%$, respectively. The performance improvement of the video packet loss by JA-MVS with a density 
of $50-500$ vehicles against the JMSR scheme is $12.9 \%$ and that against the AMVT scheme is $11 \%$. Hence, the proposed JA-MVS performs better in terms of the video packet loss ratio.

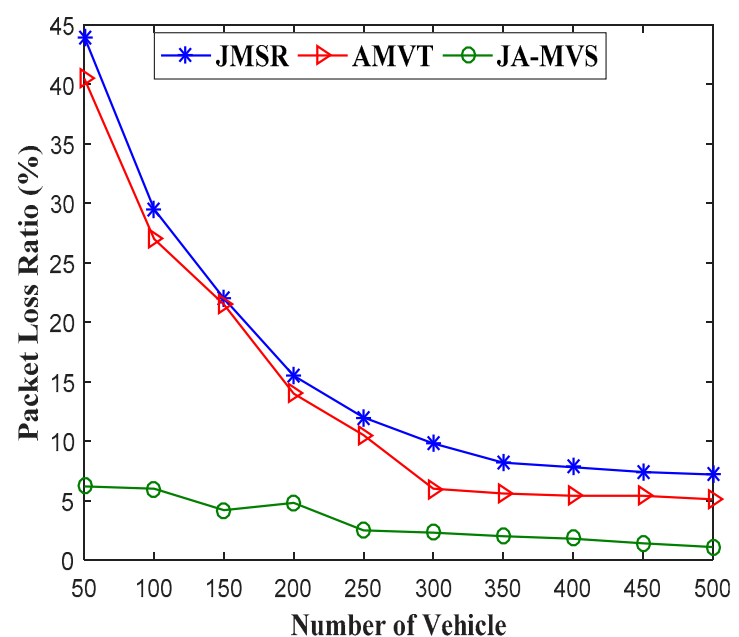

(a)

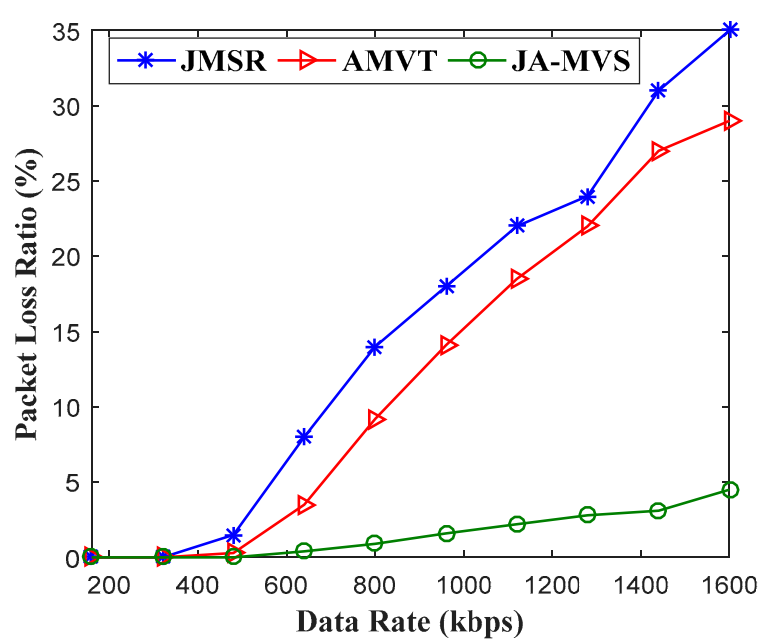

(b)

Figure 5. Packet Loss Ratio based on (a) varied vehicle densities and (b) data rates.

Figure $5 \mathrm{~b}$ depicts the results of the PLR studied alongside the various transmitted data rates. The results show that the packet loss ratio increases as the data rate increases. At 160 to $320 \mathrm{kbps}$, there is no loss of video packetw experienced; however, a packet loss starts to manifest when the data rate is $400 \mathrm{kbps}$. The packet loss increases above 30\% for JMSR and AMVT schemes as the data rate increases. However, the highest packet loss experienced for the proposed JA-MVS scheme is 5.5\% when the data rate is at $1600 \mathrm{kbps}$. Therefore, the JA-MVS outperforms the two baseline schemes. The better performance achieved by the proposed scheme is related to the reliable selection of NFV based on the direction and position of the C-NFV at the junctions of the road. The aforementioned considered parameters have helped in attaining successful video packet delivery with fewer packet losses. The average percentage gains of the three schemes are $15.4 \%, 15.5 \%$ and $2.1 \%$ for JMSR, AMVT, and JA-MVS, respectively. The average percentage gains of the performance of JA-MVS against JMSR and AMVT are $13.3 \%$ and $13.4 \%$, respectively.

The SSIM is measured as a value between 0 and 1; the results are presented in Figure $6 \mathrm{a}, \mathrm{b}$ accordingly. Figure 6a depicts the results obtained based on the SSIM index of the video transmission in relation to various vehicle densities. Based on the results obtained, the SSIM index increases gradually for both the three schemes as the vehicle density increases. The highest video SSIM index values were observed when the vehicle density is between 300 and 350. For the JMSR scheme, the SSIM index increases gradually until it starts to decline when the vehicle density is above 350; however, despite the fall in the video quality, the result is above the average value of the SSIM index of 0.5. Further, the SSIM index of the AMVT scheme also gradually increases as the number of vehicles increases; the increase almost became static with a small increase when the number of vehicles was 200 to 500 . The SSIM index of the proposed JA-MVS scheme increases based on the increase in vehicle density. The proposed JA-MVS scheme performs better than the two baseline schemes in terms of the SSIM index. The observed increase in the SSIM index is connected to the approach used for the selection of the NFV in the junction area of the road. This approach avoids the drop of video packets because vehicles that are not moving towards the DVN and that do not have the required link quality are not considered. In addition, the approach avoids the occurrence of loops or local maxima as opposite direction vehicles are avoided, except in the case of the non-availability of a vehicle in the direction of the DVN. Consequently, the results show that JA-MVS outperforms both JMSR and AMVT. The percentage gains of the three schemes are $77.6 \%, 81.0 \%$ and $84.7 \%$ for JMSR, AMVT and the proposed JA-MVS schemes, respectively. 
The average percentages of performance improvement of the JA-MVS over JMSR and AMVT are 7.1\% and $3.7 \%$, respectively.

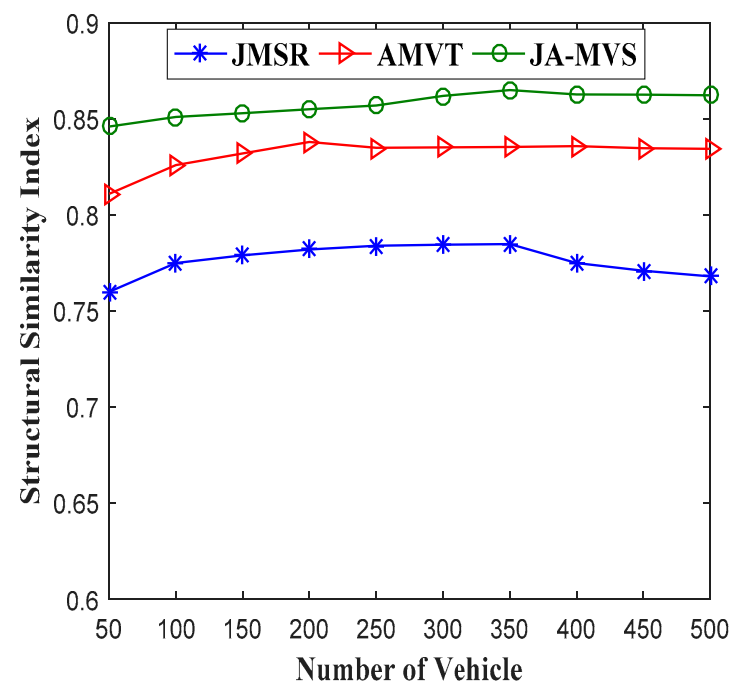

(a)

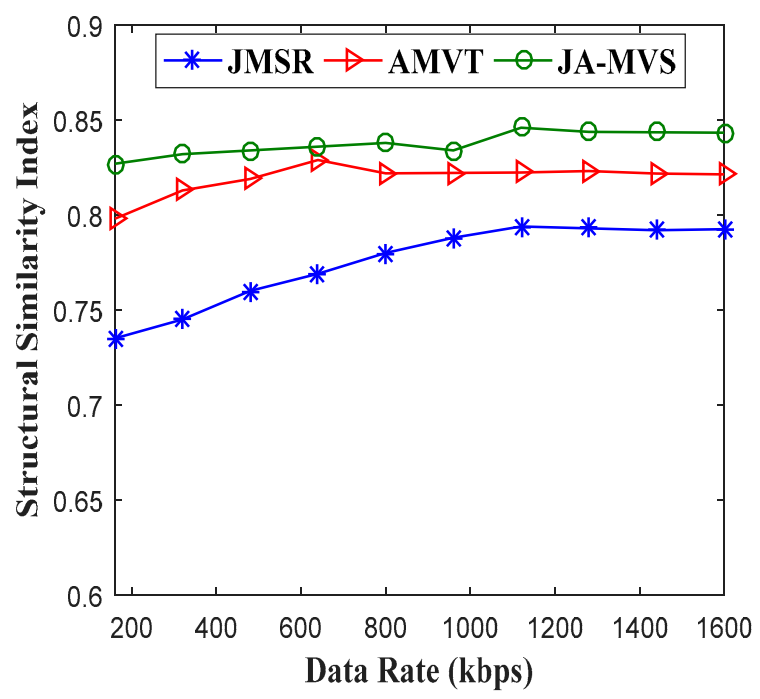

(b)

Figure 6. Structural Similarity Index based on (a) varied numbers of vehicles and (b) data rates.

Figure $6 \mathrm{~b}$ depicts the results of the SSIM index based on different video data rates. The different data rates of transmission are employed in order to show that the proposed scheme has the ability to make a fast NFV selection for video data transmission without encountering a large queuing of video data packets at the receiver vehicle. The result of the experimentation demonstrates that the value of the SSIM index increases as the transmitted data rates increases for all the three schemes. The increase in the value of the SSIM index reaches its peak when the data rate is above $1000 \mathrm{kbps}$ and below $1400 \mathrm{kbps}$. The SSIM index value tends to decline when the data rate is above $1400 \mathrm{kbps}$. However, the values of the SSIM index for each of the schemes are above average value, which is 0.5. Further, as observed from the results, the proposed JA-MVS performs better than both JMSR and AMVT. The improved performance can be related to the comprehensive selection procedure which includes all three different cases of the junction area, which provides a faster and more current status of vehicle position before selecting a vehicle as an NFV. Considering the selection, video traffic due to video queuing is minimized; hence, there is little or no traffic of video data. Considering the results, the proposed JA-MVS scheme outperforms the two baseline schemes. The average percentage gains of the three schemes are $77.5 \%, 80.1 \%$ and $84.0 \%$ for JMSR, AMVT, and the proposed JA-MVS, respectively. The percentages of the performance improvement of JA-MVS against JMSR and AMVT are $6.5 \%$ and $3.9 \%$, respectively.

The End-to-End Delay (E2ED) is studied alongside different densities of vehicles and data rates, which are shown in Figure 7a,b, respectively. Figure 7a demonstrates that both JMSR and JA-MVS have lower transmission delay in contrast with AMVT. The E2ED has been plotted alongside different densities of vehicles. A high delay is experienced in AMVT considering the fact that more than two routes have been considered, which can lead to the severe collision of video packets due to route coupling, thus causing a delay in video packet arrival time. Although, the JMSR also encountered a little delay, it is lower than that of AMVT. The result of the E2ED of JMSR is almost the same as that of the proposed JA-MVS scheme. This is connected with the fact that there is frequent signaling during communication between all neighbor vehicles. In video streaming, the delivery of data packets with few losses is more important than a delay of $\leq 5 \mathrm{~s}$ being experienced, because the loss of packets affects the quality of the video streaming. Thus, there is a balance in the tradeoff between cost and performance. Meanwhile, the overall delay experienced for the two aforementioned schemes is minimal. Therefore, the JA-MVS scheme outperformed the JMSR with only a small marginal difference. The JA-MVS 
performs better than the AMVT scheme because the proposed scheme employed different junction area situations to select the best forwarding vehicle towards the DVN. It also continues transmission through the selected NFV except if the vehicle is no longer in the neighborhood of the PFV. The percentage of E2ED encountered is computed considering the maximum allowable delay of $5 \mathrm{~s}$. Thus, the JA-MVS, JMSR, and AMVT schemes obtain values of $2.7 \%, 2.9 \%$, and $14.0 \%$, respectively. The percentages of performance improvement of the JA-MVS against JMSR and AMVT are $2.0 \%$ and $11.3 \%$, respectively.

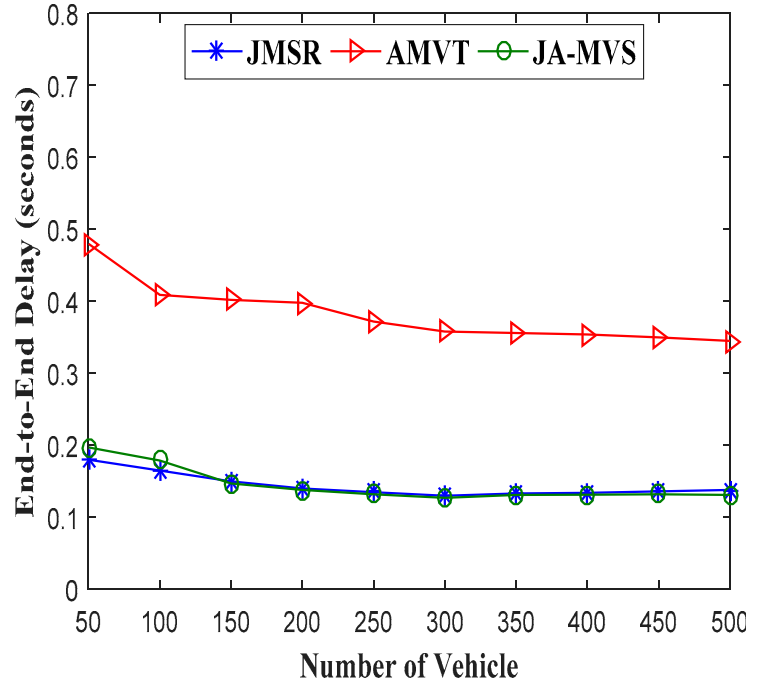

(a)

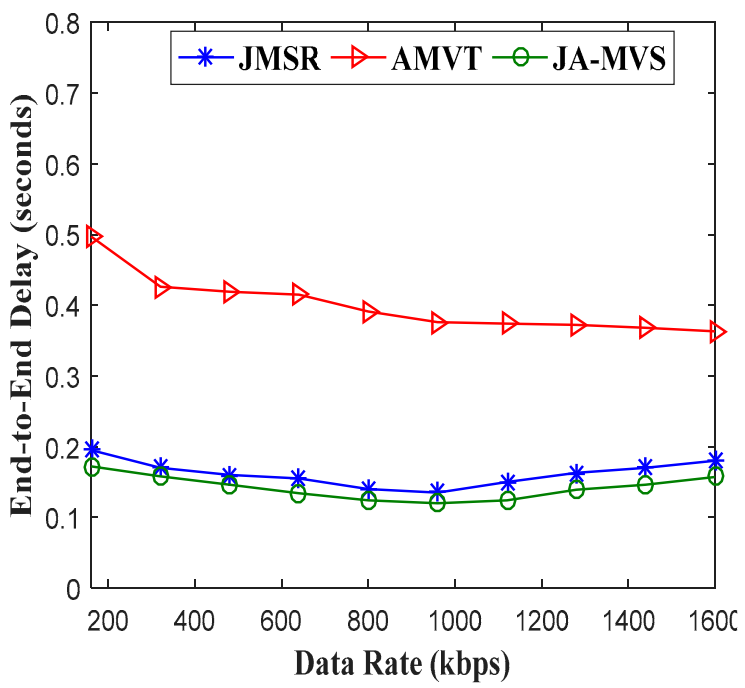

(b)

Figure 7. End-to-End Delay based on (a) varied numbers of vehicles and (b) data rates.

Figure $7 \mathrm{~b}$ depicts the E2ED results plotted alongside different data rates in order to assess the performance of the proposed scheme. The proposed JA-MVS has the lowest delay compared to both the JMSR and AMVT schemes. The high delay experienced in AMVT is connected to the high data rate transmitted, which causes queuing of video packets at the transmitting vehicle because of route coupling, which causes a collision. The JMSR and the proposed JA-MVS schemes have an almost equal delay. However, JMSR encounters higher video packet loss. Nevertheless, JA-MVS outperformed the JMSR scheme because it takes into consideration junctions with vehicles moving in opposite directions in the case of the non-availability of a vehicle in the direction of the DVN. The proposed scheme performed better than the AMVT because JA-MVS employs only two paths for the video streaming forwarding, while AMVT utilizes more than two paths, which leads to video packet collision. The average percentage delay encountered is considered based on the maximum allowable delay of $5 \mathrm{~s}$. The JA-MVS, JMSR and AMVT schemes obtain values of $2.9 \%, 3.2 \%$, and $10.9 \%$, respectively. The percentages of performance improvement of JA-MVS against JMSR and AMVT are $0.3 \%$ and $8.0 \%$, respectively.

\section{Conclusions}

In this paper, a junction-aware vehicle selection strategy for multipath video streaming in a vehicular network has been proposed. The JA-MVS scheme transmits video packets considering the different positions of the on-road junctions and the SINR as the signal quality for best forwarding vehicle selection. In the junction-aware algorithm, a vehicle that is ahead of the junction and moving toward the DVN is given higher priority to establish reliable video packet forwarding in the junction area. The SINR is an important metric for evaluating vehicle signal strength considering the urban scenario, which has a lot of obstacles that affect vehicle signal during transmission. The simulation results validate that the JA-MVS scheme significantly improves the video transmission performance in relation to the increase in quality of the video streaming, with a lower PLR and higher SSIM and a 
decrease in the overall E2ED of the video packet transmission. In addition, the simulation shows that the overall performance of the JA-MVS outperformed the two baseline schemes of JMSR and AMVT. Moreover, to further extend the proposed scheme, future research should focus on different kinds of roads including highway bridges and bent roads, considering their effects on video data packet forwarding to achieve quality video streaming in VANETs.

Author Contributions: Conceptualization, A.A. and A.H.A.; methodology, A.H.A.; software, I.A.; validation, H.E.-S., J.L. and M.P.; formal analysis, A.A.; investigation, I.A.; resources, A.H.A.; data curation, H.E.-S.; writing—original draft preparation, A.A.; writing—review and editing, M.P.; visualization, J.L.; supervision, A.H.A.; project administration, I.A.

Acknowledgments: This research was supported by the Roadway, Transportation, and Traffic Safety Research Center (RTTSRC) of the United Arab Emirates University (grant number 31R151) and in part by the Science and Technology Program of Guangzhou under Grant 201704030133, A Knowledge-Connection and Cognitive-Style-based Mining System for Massive Open Online Courses and Its Application (PRO16-1300).

Conflicts of Interest: The authors declare no conflict of interest.

\section{References}

1. Kaiwartya, O.; Abdullah, A.H.; Cao, Y.; Altameem, A.; Prasad, M.; Lin, C.T.; Liu, X. Internet of vehicles: Motivation, layered architecture, network model, challenges, and future aspects. IEEE Access 2016, 4, 5356-5373. [CrossRef]

2. Sheet, D.K.; Kaiwartya, O.; Abdullah, A.H.; Cao, Y.; Hassan, A.N.; Kumar, S. Location information verification using transferable belief model for geographic routing in vehicular ad hoc networks. IET Intell. Transp. Syst. 2016, 11, 53-60. [CrossRef]

3. Kaiwartya, O.; Kumar, S. Geocasting in vehicular adhoc networks using particle swarm optimization. In Proceedings of the International Conference on Information Systems and Design of Communication, Lisbon, Portugal, 16-17 May 2014; pp. 62-66.

4. Qureshi, K.N.; Abdullah, A.H.; Kaiwartya, O.; Ullah, F.; Iqbal, S.; Altameem, A. Weighted link quality and forward progress coupled with modified RTS/CTS for beaconless packet forwarding protocol (B-PFP) in VANETs. Telecommun. Syst. 2016, 1-16. [CrossRef]

5. Kaiwartya, O.; Kumar, S. Enhanced caching for geocast routing in vehicular Ad Hoc network. In Intelligent Computing, Networking, and Informatics; Springer: New Delhi, India, 2014; pp. 213-220.

6. Cao, Y.; Song, H.; Kaiwartya, O.; Zhou, B.; Zhuang, Y.; Cao, Y.; Zhang, X. Mobile edge computing for big-data-enabled electric vehicle charging. IEEE Commun. Mag. 2018, 56, 150-156. [CrossRef]

7. Wang, L.; Yang, H.; Qi, X.; Xu, J.; Wu, K. iCast: Fine-Grained Wireless Video Streaming Over Internet of Intelligent Vehicles. IEEE Internet Things J. 2019, 6, 111-123. [CrossRef]

8. Aliyu, A.; Abdullah, A.H.; Kaiwartya, O.; Cao, Y.; Lloret, J.; Aslam, N.; Joda, U.M. Towards video streaming in IoT Environments: Vehicular communication perspective. Comput. Commun. 2018, 118, 93-119. [CrossRef]

9. Cao, Y.; Wang, T.; Zhang, X.; Kaiwartya, O.; Eiza, M.H.; Putrus, G. Toward anycasting-driven reservation system for electric vehicle battery switch service. IEEE Syst. J. 2018, 13, 906-917. [CrossRef]

10. Qiao, J.; He, Y.; Shen, X.S. Improving video streaming quality in 5G enabled vehicular networks. IEEE Wirel. Commun. 2018, 25, 133-139. [CrossRef]

11. Qureshi, K.N.; Abdullah, A.H.; Kaiwartya, O.; Iqbal, S.; Butt, R.A.; Bashir, F. A Dynamic Congestion Control Scheme for safety applications in vehicular ad hoc networks. Comput. Electr. Eng. 2018, 72, 774-788. [CrossRef]

12. Cao, Y.; Kaiwartya, O.; Wang, R.; Jiang, T.; Cao, Y.; Aslam, N.; Sexton, G. Toward efficient, scalable, and coordinated on-the-move EV charging management. IEEE Wirel. Commun. 2017, 24, 66-73. [CrossRef]

13. Hossain, T.; Cui, Y.; Xue, Y. Vanets: Case study of a peer-to-peer video conferencing system. In Proceedings of the 2009 6th IEEE Consumer Communications and Networking Conference, Las Vegas, NV, USA, 10-13 January 2009; pp. 1-2.

14. Rao, R.S.; Soni, S.K.; Singh, N.; Kaiwartya, O. A probabilistic analysis of path duration using routing protocol in VANETs. Int. J. Veh. Technol. 2014, 2014, 495036. [CrossRef] 
15. Kaiwartya, O.; Abdullah, A.H.; Cao, Y.; Lloret, J.; Kumar, S.; Shah, R.R.; Prasad, M.; Prakash, S. Virtualization in wireless sensor networks: Fault tolerant embedding for internet of things. IEEE Internet Things J. 2018, 5, 571-580. [CrossRef]

16. Farhan, L.; Kharel, R.; Kaiwartya, O.; Hammoudeh, M.; Adebisi, B. Towards green computing for Internet of things: Energy oriented path and message scheduling approach. Sustain. Cities Soc. 2018, 38, 195-204. [CrossRef]

17. Aliyu, A.; Abdullah, A.H.; Kaiwartya, O.; Ullah, F.; Joda, U.M.; Hassan, A.N. Multi-Path video streaming in vehicular communication: Approaches and challenges. In Proceedings of the 2017 6th ICT International Student Project Conference (ICT-ISPC), Skudai, Malaysia, 23-24 May 2017; pp. 1-4.

18. Kaiwartya, O.; Kumar, S.; Lobiyal, D.; Abdullah, A.; Hassan, A. Performance improvement in geographic routing for vehicular Ad Hoc networks. Sensors 2014, 14, 22342-22371. [CrossRef]

19. Cao, Y.; Kaiwartya, O.; Aslam, N.; Han, C.; Zhang, X.; Zhuang, Y.; Dianati, M. A trajectory-driven opportunistic routing protocol for VCPS. IEEE Trans. Aerosp. Electron. Syst. 2018, 54, 2628-2642. [CrossRef]

20. Xie, H.; Boukerche, A.; Loureiro, A.A. A multipath video streaming solution for vehicular networks with link disjoint and node-disjoint. IEEE Trans. Parallel Distrib. Syst. 2015, 26, 3223-3235. [CrossRef]

21. Aliyu, A.; Abdullah, A.H.; Sikandar, A.; Joda, U.M.; Sadiq, F.I.; Ado, A. Minimizing Route Coupling Effect in Multipath Video Streaming Over Vehicular Network. In Proceedings of the International Conference on Application of Computing and Communication Technologies, Delhi, India, 9 March 2018; Springer: Berlin/Heidelberg, Germany, 2018; pp. 139-151.

22. de Felice, M.; Cerqueira, E.; Melo, A.; Gerla, M.; Cuomo, F.; Baiocchi, A. A distributed beaconless routing protocol for real-time video dissemination in multimedia VANETs. Comput. Commun. 2015, 58, 40-52. [CrossRef]

23. Kasana, R.; Kumar, S.; Kaiwartya, O.; Kharel, R.; Lloret, J.; Aslam, N.; Wang, T. Fuzzy-based channel selection for location oriented services in multichannel VCPS environments. IEEE Internet Things J. 2018, 5, 4642-4651. [CrossRef]

24. Kaiwartya, O.; Kumar, S. Geocast routing: Recent advances and future challenges in vehicular adhoc networks. In Proceedings of the 2014 International Conference on Signal Processing and Integrated Networks (SPIN), Noida, India, 20-21 February 2014; pp. 291-296.

25. Kaiwartya, O.; Kumar, S. Guaranteed geocast routing protocol for vehicular adhoc networks in highway traffic environment. Wirel. Pers. Commun. 2015, 83, 2657-2682. [CrossRef]

26. Khasawneh, A.; Latiff, M.S.B.A.; Kaiwartya, O.; Chizari, H. A reliable energy-efficient pressure-based routing protocol for underwater wireless sensor network. Wirel. Netw. 2018, 24, 2061-2075. [CrossRef]

27. Hanan, A.H.A.; Idris, M.Y.; Kaiwartya, O.; Prasad, M.; Shah, R.R. Real traffic-data based evaluation of vehicular traffic environment and state-of-the-art with future issues in location-centric data dissemination for VANETs. Digit. Commun. Netw. 2017, 3, 195-210. [CrossRef]

28. Darwish, T.; Bakar, K.A. Traffic aware routing in vehicular ad hoc networks: Characteristics and challenges. Telecommun. Syst. 2016, 61, 489-513. [CrossRef]

29. Qureshi, K.N.; Abdullah, A.H.; Lloret, J.; Altameem, A. Road-Aware Routing Strategies for Vehicular Ad Hoc Networks. Int. J. Distrib. Sens. Netw. 2016, 12, 6.

30. Yaqub, M.A.; Ahmed, S.H.; Kim, D. Asking neighbors a favor: Cooperative video retrieval using cellular networks in VANETs. Veh. Commun. 2018, 12, 39-49. [CrossRef]

31. Junior, W.L.; Rosário, D.; Cerqueira, E.; Villas, L.A.; Gerla, M. A Game Theory Approach for Platoon-Based Driving for Multimedia Transmission in VANETs. Wirel. Commun. Mob. Comput. 2018, 2018, 2414658. [CrossRef]

32. Medeiros, I.; Junior, W.L.; Rosario, D.; Cerqueira, E.; Braun, T.; Villas, L.A. A Comparative Analysis of Platoon-Based Driving Protocols for Video Dissemination over VANETs. In Proceedings of the 2018 IEEE International Conference on Communications Workshops (ICC Workshops), Kansas City, MO, USA, 20-24 May 2018; pp. 1-5.

33. Wang, M.; Xu, C.; Jia, S.; Guan, J.; Grieco, L.A. Preference-aware fast interest forwarding for video streaming in information-centric VANETs. In Proceedings of the Communications (ICC), 2017 IEEE International Conference on Communications, Paris, France, 21-25 May 2017; pp. 1-7.

34. Sermpezis, P.; Koltsidas, G.; Pavlidou, F.-N. Investigating a junction-based multipath source routing algorithm for VANETs. IEEE Commun. Lett. 2013, 17, 600-603. [CrossRef] 
35. Salkuyeh, M.A.; Abolhassani, B. An Adaptive Multipath Geographic Routing for Video Transmission in Urban VANETs. IEEE Trans. Intell. Transp. Syst. 2016, 17, 2822-2831. [CrossRef]

36. Wu, H.; Ma, H. Opportunistic routing for live video streaming in vehicular ad hoc networks. In Proceedings of the 15th International Symposium on World of Wireless, Mobile and Multimedia Networks (WoWMoM), Sydney, Australia, 19 June 2014; pp. 1-3.

37. Wang, R.; Almulla, M.; Rezende, C.; Boukerche, A. Video streaming over vehicular networks by a multiple path solution with error correction. In Proceedings of the International Conference on Communications (ICC), Sydney, Australia, 10-14 June 2014; pp. 580-585.

38. Al-Ani, A.; Seitz, J. QoS-aware routing for video streaming in multi-rate Ad hoc Networks. In Proceedings of the 9th IFIP Wireless and Mobile Networking Conference (WMNC), Colmar, France, 11-13 July 2016; pp. 193-198.

39. Quadros, C.; Santos, A.; Gerla, M.; Cerqueira, E. QoE-driven dissemination of real-time videos over vehicular networks. Comput. Commun. 2016, 91, 133-147. [CrossRef]

40. Lai, W.K.; Tai, C.K.; Wu, T.Y.; Anpalagan, A.; Chen, J.Z. PBMP: Priority-based multi-path packet routing for vehicular ad hoc network system in city environment. Trans. Emerg. Telecommun. Technol. 2016, 27, 1331-1344. [CrossRef]

41. Dosselmann, R.; Yang, X.D. A Formal Assessment of the Structural Similarity Index; University of Regina, Department of Computer Science: Regina, SK, Canada, 2008.

42. Piñol, P.; López, O.; Martínez, M.; Oliver, J.; Malumbres, M.P. Modeling video streaming over VANETs. In Proceedings of the 7th ACM Workshop on Performance Monitoring and Measurement of Heterogeneous Wireless and Wired Networks, Kaliningrad, Russia, 4-6 September 2012; pp. 7-14.

43. Immich, R.; Cerqueira, E.; Curado, M. Shielding video streaming against packet losses over VANETs. Wirel. Netw. 2016, 22, 2563-2577. [CrossRef]

44. Yang, Z.; Li, M.; Lou, W. Codeplay: Live multimedia streaming in vanets using symbol-level network coding. In Proceedings of the 18th IEEE International Conference on Network Protocols, Kyoto, Japan, 5-8 October 2010; pp. 223-232.

45. Zaidi, S.; Bitam, S.; Mellouk, A. Hybrid error recovery protocol for video streaming in vehicle ad hoc networks. Veh. Commun. 2018, 12, 110-126. [CrossRef]

(C) 2019 by the authors. Licensee MDPI, Basel, Switzerland. This article is an open access article distributed under the terms and conditions of the Creative Commons Attribution (CC BY) license (http://creativecommons.org/licenses/by/4.0/). 